\title{
ON THE COHOMOLOGY OF $H$-SPACES OF EXCEPTIONAL TYPE
}

\author{
BY JAMES P. LIN
}

Communicated by S. Eilenberg, November 3, 1975

Since the 1950's, Araki [1], Borel [3], Bott [4], Cartan, and others investigated homological and homotopical properties of Lie groups. Using the differentiable structure of a Lie group, Araki [1] and Borel [3] calculated the $\bmod p$ cohomology rings of the exceptional groups. In this note, we indicate that many of their results can be generalized to finite $H$-spaces. In particular, the proofs of these new results will be completely independent of the existence of an infinitesimal Lie structure.

Let $G$ be a simply connected Lie group. Borel [3] used the classification theorem for simple Lie groups to prove that $H_{*}(G ; Z)$ has no $p$-torsion for $p \geqslant 7$. The following result follows from a homological argument:

THEOREM 1. Let $X$ be a simply connected finite H-space. Suppose $H^{*}(X ; Q)$ is isomorphic as algebras to the rational cohomology of an exceptional Lie group. Then $H_{*}(X ; Z)$ has no $p$-torsion for $p \geqslant 7$ and has 3 or 5 torsion of order at most 3 or 5 .

Theorem 1 is not true in general for finite simply connected $H$-spaces. John Harper (unpublished) recently discovered finite simply connected $H$-spaces whose integral homology has $p$-torsion for any odd prime $p$.

Let $G$ be a simply connected Lie group. One of the key results used to compute the homology of Lie groups is Bott's result that the homology of the loops on $G, H_{*}(\Omega G ; Z)$, has no torsion. Bott uses Morse Theory [4] to prove this result. We prove the following analogous result for $H$-spaces:

THEOREM 2 Let $X$ be a simply connected finite $H$-space with $H^{*}(X ; Q)$ isomorphic as algebras to the rational cohomology of an exceptional Lie group. Then $H_{*}(\Omega X ; Z)$ has no odd torsion.

More recently, Hodgkin [5] and Araki [2] proved that for $G$ a simply connected Lie group, $K^{*}(G ; Z)$ is torsion free. Their proofs depend heavily on the differential structure of a Lie group. Hodgkin uses the classification theorem and results of Borel and Araki on the cohomology of Lie groups. Araki uses the existence of a maximal torus.

The following result can be proven using homological methods:

AMS (MOS) subject classifications (1970). Primary 57F10, 57F25; Secondary 57F05, $55 \mathrm{G} 20,55 \mathrm{D} 45$. 
THEOREM 3. Let $X$ be a simply connected finite $H$-space with $H^{*}(X ; Q)$ isomorphic as algebras to the rational cohomology of an exceptional Lie group. Then $K^{*}(X ; Z)$ has no odd torsion.

Details and proofs will appear elsewhere.

\section{REFERENCES}

1. S. Araki, Differential Hopf algebras and the cohomology mod 3 of the compact exceptional groups $E_{7}$ and $E_{8}$, Ann. of Math. (2) 73 (1961), 404-436. MR 23 \#A1372.

2. - Hopf structures attached to K-theory: Hodgkin's theorem, Ann. of Math. (2) 85 (1967), 508-525. MR 35 \#3653.

3. A. Borel, Topology of Lie groups and characteristic classes, Bull. Amer. Math. Soc. 61 (1955), 397-432. MR 17, 282.

4. R. Bott, On torsion in Lie groups, Proc. Nat. Acad. Sci. U.S.A. 40 (1954), 586588. MR 16, 12 .

5. L. Hodgkin, On the K-theory of Lie groups, Topology 6 (1967), 1-36. MR 35 \#4950.

6. J. P. Lin, On the H-spaces with finitely generated cohomology algebras, Thesis, Princeton University, 1974.

DEPARTMENT OF MATHEMATICS, UNIVERSITY OF CALIFORNIA AT SAN DIEGO, LA JOLLA, CALIFORNIA 92037 